\title{
Anti-HER2-CAR Autologous CMV-Specific Cytotoxic T-Lymphocytes
}

National Cancer Institute

\section{Source}

National Cancer Institute. Anti-HER2-CAR Autologous CMV-Specific Cytotoxic TLymphocytes. NCI Thesaurus. Code C91091.

Autologous human cytomegalovirus (CMV)-specific human cytotoxic T-lymphocytes (CTLs) transduced with a retroviral vector encoding a human anti-Her-2 (epidermal growth factor receptor 2 ) chimeric $\mathrm{T}$ cell receptor (CAR) gene with potential immunostimulatory and antineoplastic activities. Autologous CT Ls from a patient with Her-2- and CMV-positive glioblastoma multiforme (GBM) are genetically modified to express CAR gene specific for Her-2 on their cell surfaces. After expansion in culture and reintroduction into the patient, the anti-HER2-CAR autologous CMV-specific CT Ls bind to Her-2 antigen on tumor cell surfaces; subsequently, Her-2-positive tumor cells and stem cells may be lysed. Her-2 (ErbB-2), a receptor tyrosine kinase (RT K) overexpressed by a variety of cancer cell types, plays key roles in tumor cell proliferation and tumor angiogenesis. CMV is present in the majority of GBM tumors. 